\title{
In vitro Toxicity Study of Reconstituted Amphotericin B - Lipid Derivatives Dry Powder for Nebulization
}

\author{
Kajiram Adhikari \\ Department of Pharmacology, People's Dental College and Hospital Pvt. Ltd., \\ affiliated with Tribhuvan University, Naya Bazar, Kathmandu, Nepal
}

(Received: April 06, 2016; Accepted: June 08, 2016; Published (web): December 27, 2016)

\begin{abstract}
The aim of the present research work was to evaluate the safety of reconstituted dry powder amphotericin B (AmB) inhalation via nebulizer. This study was carried out on respiratory cell lines (A549, Calu-3, NR 8383), kidney cells (293T/17), human red blood cells (RBC) and aerosol properties were determined by Andersen Cascade Impactor (ACI). AmB, a lipid derivative reconstituted dry powder was formulated by lyophilization and reconstituted into distilled water at AmB concentration at $4 \mathrm{mg} / \mathrm{ml}$. The value of MMAD, FPF were obtained as 1.7 to $2.05 \mu \mathrm{m}$ and 70 to $80 \%$, respectively. The cytotoxicity test carried out by MTT assay of lipid formulations revealed a very low toxicity on respiratory cell lines such as kidney cells, than pure AmB at concentration 1 to $8 \mu \mathrm{g} / \mathrm{ml}$ of AmB. In-vitro cytotoxicity results showed less toxicity to human red blood cells (RBC) than pure $\mathrm{AmB}$ at concentration 1 to $8 \mu \mathrm{g} / \mathrm{ml}$ of $\mathrm{AmB}$.
\end{abstract}

Key words: Amphotericin B, Cytotoxicity, Lipid drug carriers, Alveolar macrophage, Nebulizer.

\section{INTRODUCTION}

Amphotericin B (AmB), natamycin and nystatin are three main compounds of antifungal polyenes group. Among them, AmB is the drug of choice because of board spectrum and least resistance to treatment for life threatening systemic fungal infections (aspergillosis, candidiasis and cryptococcosis) including those newly emerging and azole-resistant pathogens in neutropenic patients. ${ }^{1}$ Invasive aspergillosis is the prime causes of death observed in immunocompromised patients. ${ }^{2} \mathrm{AmB}$ acts by increasing the permeability of the fungal cell membrane by binding to ergosterol components and it interacts with cholesterol in human cell membranes that causes manifestation of toxic effects and preliminary termination of treatment. ${ }^{1}$ It can cause nephrotoxicity and other related toxicities (fever,

Correspondence to: Kajiram Adhikari

Tel.: +977-01-4390105; Fax: +977-01-4390692

Email:kajiadhikari@gmail.com

Dhaka Univ. J. Pharm. Sci. 15(2): 127-134, 2016 (December) chills, vomiting, hemolysis) when it is given intravenously. ${ }^{3}$ Lipid based formulations have been developed to improve the therapeutic index and reduce the toxicity of $\mathrm{AmB}$, for example Amphocil ${ }^{\circledR}$, Ambisome $^{\circledR}$ and Abelcet ${ }^{\mathbb{R}}$, although, its limited uses due to unaffordable cost of the products. ${ }^{4}$ Water soluble reconstituted dry powder of lipid derivatives is very useful in the treatment of systemic fungal infection. Research concerning a novel delivery system for AmB with lipid nanoshperes may render a safer, more efficacious and affordable form of the drug in near future. ${ }^{5}$ An ideal drug delivery system should provide a targeted therapy that will allow effective concentrations of a drug to the site of disease without exposing others tissues to toxicity. For fungal lung infections the appropriate route of drug administration is to directly deliver the drug into lung, which has been successfully done with a nebulizer. ${ }^{6}$ Lung delivery provides targeted therapy at the infected area and reduces kidney toxicity from exposure to high concentrations of AmB. ${ }^{7}$ To reduce 
these adverse effects, nebulizer is an attractive option since high local drug concentrations are achieved with minimal systemic exposure. ${ }^{8}$ The advantages of jet nebulizers are inexpensive and do not require trained personnel. Thus our aim of this study was to evaluate aerosol properties and safety of inhalation via nebulization. This study employed lipid derivatives to formulate AmB-lipids system, which was transformed to dry powder by lyophilization. Five lipid derivatives were sodium deoxycholate (SDC), sodium deoxycholate sulfate (SDCS), sodium cholate (SC), potassium deoxycholate (KDC) and potassium cholate $(\mathrm{KC})$. AmB lipid molar ratio was $2: 1$. The depositions of drug in-vitro with delivery by jet nebulizer were determined by the Andersen Cascade Impactor (ACI). The mass median aerodynamic diameters (MMAD) of particles were 1.7- $2.05 \mu \mathrm{m}$. The Fine particle fractions $(\mathrm{FPF}<4.7$ $\mu \mathrm{m})$ of the all formulations were 70 to $80 \%$. In-vitro toxicity studies on human lung adenocarcinoma cell lines (A549), human bronchial epithelial cells (calu3), alveolar macrophage cell lines (AMs) NR8383 and kidney cells (293T/17), hemolysis were carried out to ensure that the AmB-lipid formulation was safe to those cell lines. After nebulization, the particle size of five formulations was determined by mass median aerodynamic diameter (MMAD). The aerosolization testing was employed to confirm the possibility of producing the correct dosage with the aid of a nebulizer.

\section{METERIALS AND METHODS}

\section{Materials:}

AmB was obtained from Ambalal Sarabhai, Enterprises (Vadodara, India). deoxycholic acid (DCA), sodium deoxycholate (SDC), cholic acid (CA) and sodium cholate (SC) were purchased from Sigma-Aldrich (St. Louis, USA). Potassium cholate $(\mathrm{KC})$, potassium deoxycholate (KDC) and sodium deoxycholate sulfate (SDCS) were synthesized in the laboratory. Dimethyl sulfoxide was from Riedel-de Haean, Germany. Polyamide membranes with a pore size of $0.22 \mu \mathrm{m}$ were obtained from Sartorius, Gottingen, Germany. All chemicals were of laboratory grade and used without any further purification.

\section{Methods}

Preparation of Amphotericin B-lipid formulations (AmB-KC, AmB-KDC, AmB-SC, AmB-SDC and AmB-SDCS). Sodium deoxycholate sulfate (SDCS, $245 \mathrm{mg}$ ) was taken in beaker $(100 \mathrm{ml})$ containing $30 \mathrm{ml}$ distilled water and sodium hydroxide $(2.7 \mathrm{ml}, 0.2 \mathrm{M})$ was added to this solution with constant stirring (500 rpm) in a magnetic stirrer Heidolph MR Hei-Mix L (Helodolph Instruent, Schwabach, Germany). After obtaining a clear solution, amphotericin B powder was slowly added $(\mathrm{AmB}, 250 \mathrm{mg})$ in part wise. When $\mathrm{AmB}$ was dissolved completely, then it formed a clear yellowish color solution at room temperature. Then the $\mathrm{pH}$ of the solution was adjusted by adding phosphoric acid $(0.2 \mathrm{M})$ to obtain a $\mathrm{pH}$ of 7.4 for an in situ phosphate buffer using $\mathrm{pH}$ meter (Precisa $\mathrm{pH}$ 900, Dietikon, Switzerland). The final volume of the solution was made to $50 \mathrm{ml}$ by adding distilled water. The solution was filled into $10 \mathrm{ml}$ on each vial and lyophilization by a freeze dryer (Dura Dry ${ }^{\mathrm{TM}} \mathrm{MP}$, FTS Systems Inc., NY, USA). Thus a yellowish powdered cake was formed. A similar methodology was employed to prepare sodium deoxycholate (AmB-SDC), potassium deoxycholate (AmB-KDC), potassium cholate (AmB-KC) and sodium cholate (AmB-SC) formulations as that of deoxycholic acid as well as cholic acid and AmB. This method was adopted on CDD 2012. ${ }^{9}$

Aerosol properties of the reconstituted AmBlipid dry powders. Around $50 \mathrm{mg}$ of AmB-lipid derivatives (AmB-KC, AmB-KDC, AmB-SC, AmBSDCS and AmB-SDC) of lyophilized dry powder (i.e., equivalent to $25 \mathrm{mg}$ of $\mathrm{AmB}$ ) was reconstituted with $6 \mathrm{ml}$ filtered distilled water $(4 \mathrm{mg} / \mathrm{ml}$ of $\mathrm{AmB})$ for nebulization. This $6 \mathrm{ml}$ solution was poured into a reservoir of a jet-nebulizer (Westmed Inc., Arizona, USA) and connected to a compressed nitrogen gas cylinder and the gas flow was adjusted to $8 \mathrm{~L} / \mathrm{min}$. Then the mouthpiece of the jet nebulizer was connected to an eight stage Andersen Cascade 
Impactor (ACI), (Atlanta, GA, USA). The ACI was operated at a vacuum flow rate of $28.3 \mathrm{~L} / \mathrm{min}$. First, the nebulizer was operated for one min and the aerosol generated was directed into a fume hood. Following this nebulization period, the nebulizer was operated for a $2 \mathrm{~min}$ period to the ACI. The mass median aerodynamic diameter (MMAD) was calculated during the 1-3 min nebulization time interval. The fine particle fraction was calculated from the AmB deposited on each stage from 1 to 7 . All the nebulization was carried out at room temperature to avoid any temperature effects on the deposition of the particles. Five experiments were conducted on each formulation. The drug deposited on each of the stages ( $0-7$ stages) and the metal inlet of the ACI was extracted by rinsing with $25 \mathrm{ml}$ of dimethyl sulfoxide and methanol (1:9 ratios $\mathrm{v} / \mathrm{v})$ solution. The drug deposited on each stage was determined by high-performance liquid chromatography (HPLC). For the HPLC conditions, acetate buffer $(20 \mathrm{mM}, \mathrm{pH}$ at 7.2$)$ and acetonitrile $(60: 40 \mathrm{v} / \mathrm{v})$ was used as the mobile phase at a flow rate of $1 \mathrm{ml} / \mathrm{min}$. The microbondapak $\mathrm{C}_{18}$ (Phenomenex ${ }^{\circledR}$, USA $)(150 \times 4.6 \mathrm{~mm} \times$ i.d., $5 \mu \mathrm{m})$ column was the stationary phase. UV detection was carried out at a wavelength of $405 \mathrm{~nm}$.

In vitro hemolysis assay. The human red blood cells lysis was evaluated as described by Mehta et al. ${ }^{10}$ Briefly, erythrocytes (Blood Bank, Department of Pathology, Faculty of Medicine, Songklanagarind Hospital, Prince of Songkla University, Thailand) were isolated from fresh human blood, washed three times with phosphate buffer saline solution (PBS) and centrifuged at $3000 \mathrm{rpm}$ for $5 \mathrm{~min}$. Stock solutions of AmB, AmB-KC, AmB-KDC AmB-SC, $A m B-S D C$ and AmB-SDCS were added to the suspended erythrocytes and the suspension was diluted with PBS to give final $\mathrm{AmB}$ concentration in the range $1-8 \mu \mathrm{g} / \mathrm{ml}$ and a final hematocrit of $1 \%$. The solutions were incubated at $37^{\circ} \mathrm{C}$ in an incubator for $24 \mathrm{~h}$. The unlysed cells were removed by centrifugation at $3000 \mathrm{rpm}$ for $5 \mathrm{~min}$ and the hemoglobin in the supernatant was determined by its absorbance at $540 \mathrm{~nm}$. PBS without $\mathrm{AmB}$ was used as negative control and 1\% Triton X-100 (Sigma-
Aldrich, Steinheim, Germany) was used as positive control for $100 \%$ lysis. According to the equation 1, $\%$ hemolysis can be calculated as shown below:

$\%$ Hemolysis $=\left[\right.$ Abs- $\left.-\mathrm{Abs}_{0} / \mathrm{Abs}_{100}-\mathrm{Abs}_{0}\right] \times 100$ ......... Equation......1

Where: Abs is the absorbance of sample

$: \mathrm{Abs}_{0}$ is the absorbance of negative control

$: \mathrm{Abs}_{100}$ is the absorbance of positive control

The cytotoxicity assay in respiratory cell lines and kidney cells. A549, Calu-3 and AMs NR8383, kidney cells were distributed in 96-well plates at a density of $1 \times 10^{5}$ cells/well in $100 \mu \mathrm{L}$ completed medium and allowed to attach overnight. After $24 \mathrm{~h}$ at $37^{\circ} \mathrm{C}$ and $5 \% \mathrm{CO}_{2}, 95 \%$ humidity, the medium $(100 \mu \mathrm{L})$ was replaced with medium containing various concentrations of drug formulations ( 1 to 8 $\mu \mathrm{g} / \mathrm{ml})$ or an equivalent concentration. After incubation for $24 \mathrm{~h}, 50 \mu \mathrm{L}$ of a solution of MTT at $1.25 \mathrm{mg} / \mathrm{ml}$ (Invitrogen, USA) were added and incubated for a further $4 \mathrm{~h}$ at $37^{\circ} \mathrm{C}$ in an atmosphere of $5 \% \mathrm{CO}_{2}$ and $95 \%$ humidity, covered with an aluminum foil. After completion of the $4 \mathrm{~h}$ incubation, the solutions were removed from the 96 well plates and $100 \mu \mathrm{L}$ of DMSO added to dissolve the formazan crystals. The optical densities (OD) were measured at $570 \mathrm{~nm}$ in a microplate reader (Biohit BP 800, Helsinki, Finland). Control cells were incubated with medium without AmB. Four wells were used for each concentration and timepoint. The percentage of surviving cells was calculated from the following formula: ( $O D_{\text {treated }} / O D$ control $) \times 100$. Three independent experiments were done. The number of viable cells in the treated well was compared to those in the untreated well and estimated as percent viability.

Statistical analysis. Data were presented as mean \pm standard deviation (SD) from at least three samples unless indicated. The data were compared using Student's $t$ test for independent samples and by analysis of variance (ANOVA). All statistical comparisons were calculated using the SPSS software version 16.0 (SPSS Inc., Chicago, IL). A 
significance of level of $\mathrm{p}$-value $<0.05$ was considered statistically significant.

\section{RESULTS AND DISCUSSION}

Aerosol properties of reconstituted AmB-lipid formulations after nebulization. The in-vitro deposition of AmB-lipid derivatives in the Andersen Cascade Impactor (ACI) using a reconstituted solution and jet nebulization as described in the materials and method section. The properties of the aerosolized AmB-lipid derivatives (AmB-KC, AmB$\mathrm{KDC}, \mathrm{AmB}-\mathrm{SC}, \mathrm{AmB}-\mathrm{SDC}$ and AmB-SDCS, ) are presented in table 1. Part of this research related to the aerosol property of the two formulations, AmBSDCS and AmB-SDC was previously reported by Gangadhar et al. ${ }^{13}$ The physical properties of the AmB-lipid formulations are the most important factors that determine their deposition in the small airways of the lung for targeting the alveolar macrophages. The FPF was the amount of AmB smaller than $4.7 \mu \mathrm{m}$. To achieve the best result of aerosolization for delivery of the drug into a diseased lung, small aerosolized particle sizes with the MMADs that range from 1 to $5 \mu \mathrm{m}$ are required. MMADs of all the AmB-lipid formulations used in this study were between 1.70 to $2.05 \mu \mathrm{m}$ (Table 1). The percentages of the FPF of the AmB-lipid formulations were found to be between $70-80 \%$. However, the powders had very poor flow properties, so they were not suitable for use in a dry powder inhalation. For nebulization, the dry powder was reconstituted with distilled water and found to be in a highly soluble and stable form, so this solution was suitable for jet nebulization. It revealed that these formulations were stable without degradation of AmB during the nebulization process.

Table 1. Aerosol properties of reconstituted AmB-lipid dry powders with distilled water $(\mathrm{Mean} \pm \mathrm{SD}, \mathrm{n}=5$ ).

\begin{tabular}{lccc}
\hline Material & \% content & MMAD $(\mu \mathrm{m})$ & \% FPF $(<4.7 \mu \mathrm{m})$ \\
\hline AmB-SDC & $100.8 \pm 1.8$ & $1.70 \pm 0.3$ & $70 \pm 3.9$ \\
AmB-SDCS & $101.9 \pm 3.4$ & $1.74 \pm 0.4$ & $80 \pm 2.3$ \\
AmB-KDC & $100.4 \pm 0.3$ & $1.81 \pm 0.3$ & $71 \pm 4.9$ \\
AmB-KC & $100.2 \pm 0.5$ & $1.89 \pm 0.4$ & $72 \pm 4.2$ \\
AmB-SC & $99.5 \pm 0.4$ & $2.05 \pm 0.3$ & $74 \pm 3.3$ \\
\hline
\end{tabular}

In vitro hemolysis assay. Hemolysis of human red blood cells was evaluated by a reported method as described in the materials and methods section. Erythrocytes treated with AmB-KC, AmB-KDC, AmB-SC, AmB-SDCS, AmB-SDC, and AmB showed some hemolysis as shown in figure 1 . In this study, we had chosen a concentration range of 1-8 $\mu \mathrm{g} / \mathrm{ml}$ according to $\mathrm{Yu}$ et al. The safety level of hemolysis was reported by $\mathrm{Yu}$ et al. ${ }^{14}$ less than $5 \%$ of hemolysis increased as the concentration of $\mathrm{AmB}$ increased up to $17 \%$. AmB-SDCS, AmB-SDC, AmB$\mathrm{KDC}, \mathrm{AmB}-\mathrm{KC}, \mathrm{AmB}-\mathrm{SC}$ and $\mathrm{AmB}$, each at a concentration of $8 \mu \mathrm{g} / \mathrm{ml} \mathrm{AmB}$, caused some red blood cell hemolysis of $9 \%, 14 \%, 14 \%, 14 \%, 13 \%$ and $17 \%$, respectively so all AmB-lipid formulations produced less hemolysis than AmB. Among these five formulations, AmB-SDCS was least hemolysis than others. It was due to the sulfate ions interact and stabilize micelle and low release of free $\mathrm{AmB}$ and free form $\mathrm{AmB}$ is less available to formation of dimeric form, which causes for hemolysis. These lipid derivatives may be able to stabilize AmB. Forster et al. ${ }^{15}$ reported that lecithin-stabilized emulsions produced low hemolysis. They explained that the reduced hemolysis of the liposome and the lecithin-stabilized emulsions was due to the strong binding between the $\mathrm{AmB}$ and the phospholipid. Chuealee et al. ${ }^{16}$ studied various cholesterol derivatives for the formulation of $\mathrm{AmB}$ and found that $\mathrm{AmB}$ was stabilized in a similar manner and hemolysis was prevented. The hemolytic effects were effectively or significantly reduced compared to conventional surfactants such as SDC and cholesterol. 
Cytotoxicity assay. The toxicity of the lipid formulations (AmB-KC, AmB-KDC, AmB-SC, AmB-SDC and AmB-SDCS) and AmB on A549, AM NR 8383 and Calu-3 cell lines and kidney cells (293T/17) were estimated at different concentrations from 1 to $8 \mu \mathrm{g} / \mathrm{ml}$ by the MTT reduction assay after $24 \mathrm{~h}$ of exposure as shown in Figure 2. Typical effective concentration of $\mathrm{AmB}$ in the blood circulation is $1 \mu \mathrm{g} / \mathrm{ml}$ so the lung tissue should have a similar concentration. ${ }^{17,18}$ A typical testing for the $\mathrm{AmB}$ concentration in vitro was between 8-16 times, in this case we had chosen 8 times. ${ }^{14}$ The lipid formulations (AmB-KC, AmB-KDC, AmB-SC, AmB-SDC and AmB-SDCS) and AmB up to $8 \mu \mathrm{g} / \mathrm{ml}$ showed very low cytotoxicity to A549 cells. The viability of A549 cells after treatment with AmBSDCS, AmB-SDC, AmB-KDC, AmB-KC, AmB-SC and $A m B$ were 95, 91, 89, 94, 95 and 92\%, respectively (Figure $2 \mathrm{~A}$ ). For the Calu-3 cells they showed above $92 \%$ viability with all AmB formulations up to $8 \mu \mathrm{g} / \mathrm{ml}$. This indicated that the five formulations were not toxic to the lung cell lines Calu-3 (Figure 2 B). In the case of the AM NR8383 cell lines, the percentage of viability was over $80 \%$ with the AmB lipid formulations (AmB-KC, AmBKDC, AmB-SC, AmB-SDC and AmB-SDCS) at concentration of AmB up to $8 \mu \mathrm{g} / \mathrm{ml}$ and more than $99 \%$ viability was achieved at a lower concentration of $1 \mu \mathrm{g} / \mathrm{ml}$. In the case of pure AmB, the viability of AM NR8383 cells rapidly declined from 93 to 56\% with increasing concentrations of $\mathrm{AmB}$ from 1 to 8 $\mu \mathrm{g} / \mathrm{ml}$. There was more than $80 \%$ of cell viability at concentrations of AmB $1-4 \mu \mathrm{g} / \mathrm{ml}$ whereas at 8 $\mu \mathrm{g} / \mathrm{ml}$, it was drastically decreased by up to $56 \%$ (Figure $2 \mathrm{C}$ ). AmB is known as a nephrotoxic drug, so its clinical application is limited due to its major toxicity. ${ }^{19}$ The incidence of nephrotoxicity was reported 49-65\% in conventional AmB deoxycholate. ${ }^{20}$ To minimize this toxic effect, lipid derivative carriers (SDCS, KC and KDC) were synthesized in a laboratory, SDC and SC were purchased from the market and AmB-lipid formulations (AmB-KC, AmB-KDC, AmB-SC, AmB-SDC and AmB-SDCS) were prepared by lyophilization process. This toxicity test was done in- vitro study on human kidney cells (293T/17) as shown in (Figure $2 \mathrm{D}$ ). The percentage viability of kidney cells was over $90 \%$ after challenging with $\mathrm{AmB}$ or $\mathrm{AmB}$ formulations at $1 \mu \mathrm{g} / \mathrm{ml}$. A slight decrease of viability to around $83 \%$ at $2 \mu \mathrm{g} / \mathrm{ml} \mathrm{AmB}$ was observed. However, at 4 and $8 \mu \mathrm{g} / \mathrm{ml} \mathrm{AmB \text {, }}$ dramatic cell death was observed. AmB-lipid formulations (AmB-KC, AmB-KDC, AmB-SC, AmB-SDC and AmB-SDCS) provided some protection of kidney cells as viability after treatment with $4 \mu \mathrm{g} / \mathrm{ml}$ was higher than $80 \%$ and except AmBSDC, AmB-SDCS, AmB-SC, AmB-KC and AmB$\mathrm{KDC}$ formulations were higher than $80 \%$ at $8 \mu \mathrm{g} / \mathrm{ml}$. AmB-SDC at these concentrations was slightly less toxic than pure $\mathrm{AmB}$. The micelles provided a relatively weak barrier compared to the lipid bilayers of liposomes or lipid matrix or LNPs. The release of AmB from micelles may be faster than AmBisome ${ }^{\circledR}$ or AmB entrapping LNPs. ${ }^{20,20 b}$ Moreover, strong interaction between $\mathrm{AmB}$ and lipids such as phospholipids and /or cholesterol can retard release of AmB from LPNs or AmBisome ${ }^{\mathbb{R}} .^{21}$ Therefore, LPNs or liposomes that entrap drugs in their lipid matrix or bilayers are able to decrease cytotoxicity of the entrapped drugs. ${ }^{22}$ In this case it is possible that AmB-SDCS, AmB-SC, AmB-KC and AmB-KDC lipid formulations can form more stable micelles than AmB-SDC. Therefore the free AmB release from AmB-SDCS, AmB-SC, AmB-KC and AmB-KDC lipid formulations may be slower than that from AmB-SDC resulting in a lower toxicity to the kidney cells. These lipid formulations seemed to be less toxic than pure AmB. In overall toxicity studies of AmB-lipid formulations in respiratory associated three (A549, Calu-3 and NR 8383) cell lines and kidney cell lines $(293 \mathrm{~T} / 17)$, they were showed that SDCS, KDC, KC and SC formed more stable micelles than SDC. Therefore, the release of free AmB from AmB-SDCS, AmB-KDC, AmB-KC and AmB-SC may be slower than that from AmB-SDC resulting in less toxicity for all tested cells up to 8 $\mu \mathrm{g} / \mathrm{ml}$ concentration of $\mathrm{AmB}$. 


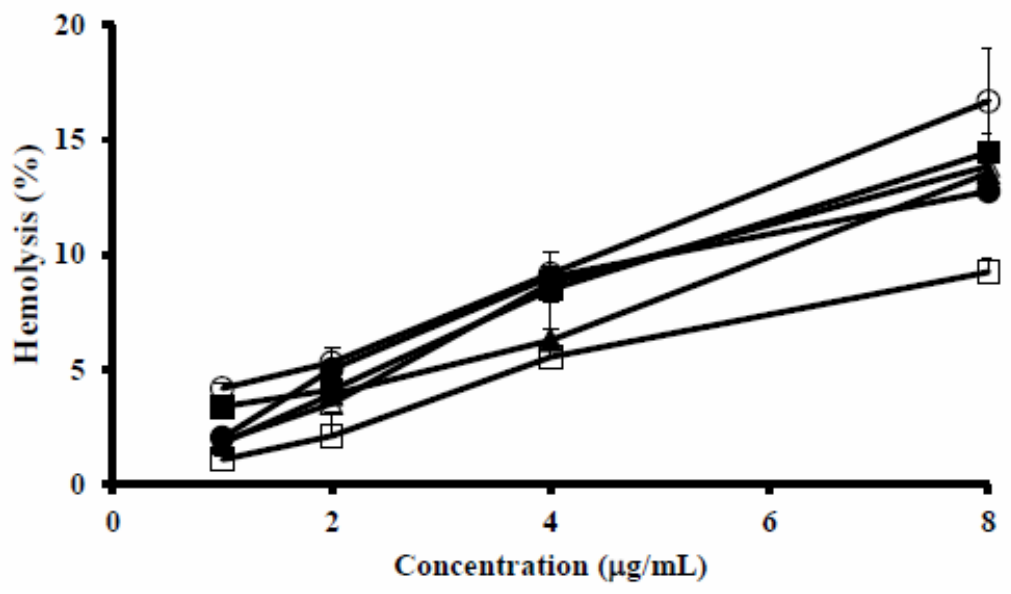

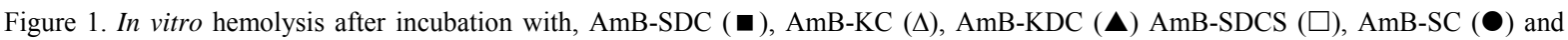
$\mathrm{AmB}(\mathrm{O})$ at $24 \mathrm{~h}($ mean $\pm \mathrm{SD}, \mathrm{n}=3)$.
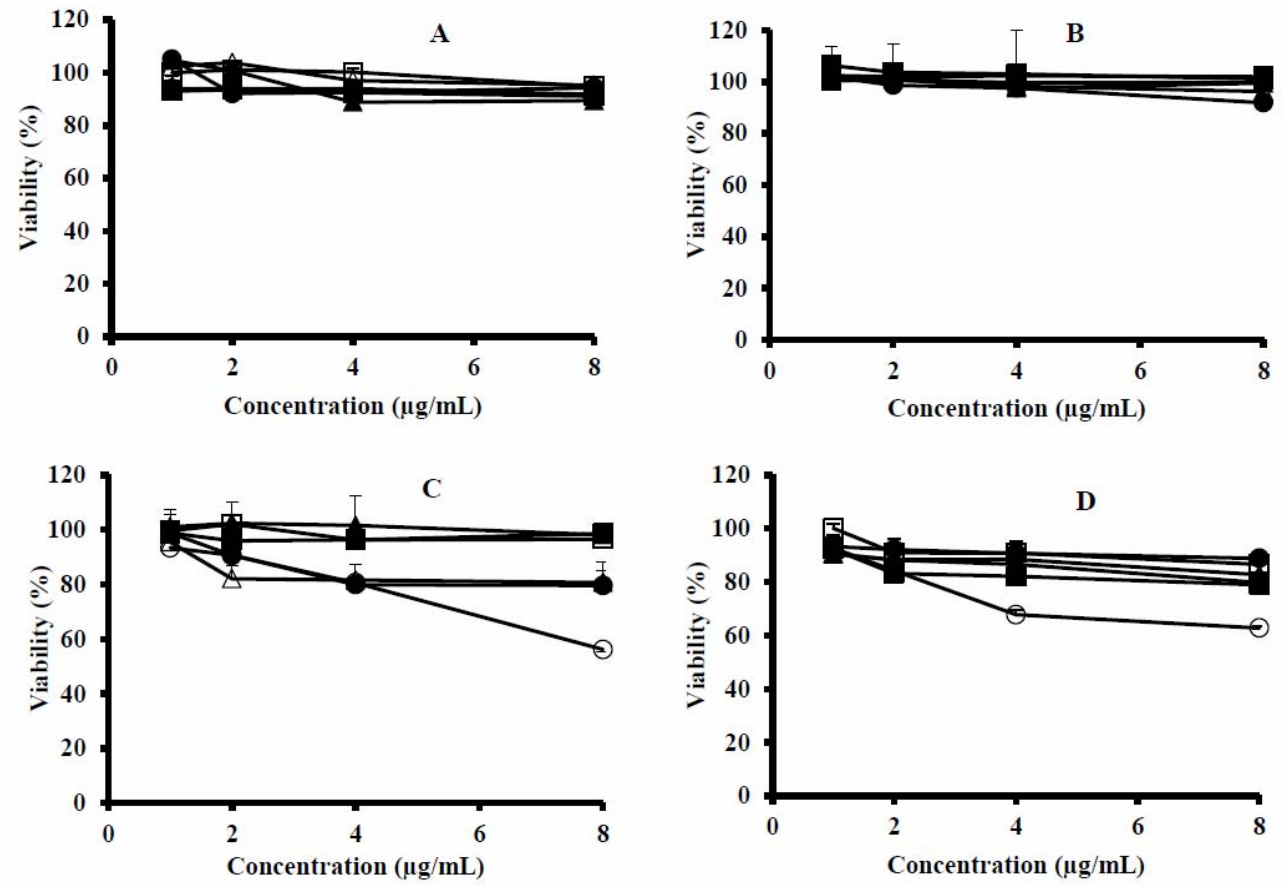

Figure 2. Viability of A549 cell line (A), calu-3 cell line (B), AMs NR8383 (C) and kidney cells (293T/17) (D) after they have been incubated with $\mathrm{AmB}(\mathrm{O}), \mathrm{AmB}-\mathrm{SDCS}(\square), \operatorname{AmB}-\mathrm{SDC}(\bullet), \operatorname{AmB}-\mathrm{KDC}(\bullet), \operatorname{AmB}-\mathrm{KC}(\Delta), \operatorname{AmB}-\mathrm{SC}(\bullet) ;(m e a n \pm \mathrm{SD}, \mathrm{n}=4)$.

\section{CONCLUSION}

Five lipid derivatives such as $\mathrm{KC}, \mathrm{KDC}, \mathrm{SC}$, SDC and SDCS, were chosen as lipid drug carriers. These carriers were applied to develop as lipid drug carriers system as a reconstituted dry powder AmBlipid formulations. AmB-lipid formulations were successfully prepared by lyophilization process (freeze drying) in mole ratio 1:2 (AmB:lipid carrier), 
formed solid caked, which was a very light, free flowing, hygroscopic in nature. Aerosolization characteristics such as MMADs were obtained ranges 1.70- $2.05 \mu \mathrm{m}$ with high FPF $70-80 \%$. These lipid drug carriers play vital role play to dissolve the poorly soluble $\mathrm{AmB}$ into highly soluble and stable solution form. The cytotoxicity examinations of respiratory associated cell lines (A549, calu-3 and AM NR 8383), kidney cells and human RBC hemolysis were shown higher viability with AmBlipid formulations as compared to pure AmB. This was possible for targeting the AM in lung fungal infections. Therefore, we carried out all the relevant study of AmB-lipid formulations and conclude that they were suitable for the treatment of pulmonary aspergillosis by targeting AM via jet nebulization. Among five AmB-lipid formulations, it can be concluded that SDCS was the best lipid drug carrier in terms of its aerosol property, toxicity tests.

\section{ACKNOWLEDGEMENT}

This research work was financially supported by the National Research University Project Grant for $\mathrm{PhD}$ Research Programs at the Prince of Songkla University, Faculty of Pharmaceutical Sciences, Department of Pharmaceutical Technology, Hat Yai, Thailand. The author would like to thanks the Drug Delivery System Excellence Centre for use of their facilities. I would like to thanks my supervisor, Assoc. Prof. Dr. Teerapol Srichana, under his supervision, I had successfully completed my $\mathrm{PhD}$ program.

Conflict of interest: The author states no conflicts of interest.

\section{References}

1. De Pouw, B.E.2000.New antifungal agents and preparations. Int. J. Anticrob. Agents. 16, 147-150.

2. Lemke, A., Kinder, A.F. and Kayser, O. 2005. Amphotericin B, Appl. Microbial.Biotechnol. 68, 151-162.

3. Antoniadou, A., and Dupont, B., 2005. Lipid formulations of amphotericin B: where are we today? J. Mycol. Med. 15, $230-238$.
4. Egger, S.S., Meier, S., Leu, C., Christein, S. and Gratwohl, A. 2009. Drug interactions and adverse events associated with antimycotic drugs used for invasive aspergillosis in hematopoietic SCT. Bone Marrow Transplant. 45, $1197-$ 1203.

5. Kirchgessner, M. 2008. Amphotericin B. J. Exot. Pet Med. 17, 54-56.

6. Drew, R. 2006. Potential role of aerosolized amphotericin B formulation in the prevention and adjunctive treatment of invasive fungal infections. Int. J. Antimicrob. Agents. 27, 3344.

7. Chuealee, R., Aramwit, P., Noipha, K. and Srichana, T. 2011. Bioactivity and toxicity studies of amphotericin B incorporated in liquid crystals. Eur. J. Pharm. Sci. 43, 308317.

8. Le, J. and Schiller, D.S.2010. Aerosolized delivery of antifungal agents: Current Fungal Infect Rep. 4, 96-102.

9. Adhikari, K.R. and Srichana, T. 2012. Formulation of amphotericin B-lipid derivatives and its evaluation. $C D D$ Proceedings. 129-132.

10. Mehta, R., Lopez, B.G., Hopfer, R., Mills, K.and Juliano, R.L. 1984. Liposomal AmB is toxic to fungal cells but not to mammalian cells. Biochem. Biophys. Acta. 770, 230-234.

11. Huttunen, K., Ruotsalainen, M., Iivanainen, E., Torkko, P., Katila, M.L. and Hirvonen, M.R. 2008. Inflammatory responses in RAW 264.7 macrophages caused by mycobacteria isolated from moldy houses. Environ Toxicol Pharmacol. 8, 237-244.

12. Punturee, K., Wild, C.P. and Vinitketkumneun, U. 2004. Thai medicinal plants modulate nitric oxide and tumor necrosis factor in J774.2 mouse macrophages. $J$. Ethnopharmacol. 95, 183-189.

13. Gangadhar, K.N., Adhikari, K.R. and Srichana, T. 2014. Synthesis and evaluation of sodium deoxycholate sulfate as a lipid drug carrier to enhance the solubility, stability and safety of an amphotericin B inhalation formulation. Int. J. Pharm. 471, 430-438.

14. Yu, B.G., Okano, T., Kataoka, K. and Kwon, G. 1998. Polymeric micelles for drug delivery: solubilization and haemolytic activity of amphotericin B. J. Control. Release. 53, 131-136.

15. Forster, D., Washington, C. and Davis, S.S. 1988. Toxicity of solubilized and colloidal amphotericin B formulations to human erythrocytes. J. Pharm. Pharmacol. 40,325-328.

16. Chuealee, R., Aramwit, P., Noipha, K. andSrichana, T. 2011. Bioactivity and toxicity studies of amphotericin B incorporated in liquid crystals. Eur. J. Pharm. Sci. 43: 308317. 
17. Bennett, J.E. 1996. Antimicrobial Agents, In: Goodman and Gillman's (Ed), The Pharmacological Basis of Therapeutics. McGraw Hill, New York, USA. pp. 1175-1190.

18. AHFS Drug Information. 2009. American Society of HealthSystem Pharmacists, Inc. 7272 Wisconsin Avenue, Bethesda, MD 20814, USA. pp. 542-554.

19. Jung, S.H., Lim, D.H., Jung, S.H., Lee, J.E., Jeong, K.S., Seong, H. and Shin, B.C. 2009. Amphotericin B-entrapping lipid nanoparticles and their in-vitro and in-vivo characteristics. Eur. J. Pharm. Sci. 37, 313-320.

20a Moribe, K., Maruyama, K. and Iwatsuru, M. 1999a. Encapsulation characteristics of nystatin in liposomes: effects of cholesterol and polyethylene glycol derivatives. Int. J. Pharm. 188, 193-202. 20b. Moribe, K., Maruyama, K. and Iwatsuru, M. 1999 b. Molecular localization and state of amphotericin B in PEG liposomes. Int. J. Pharm. 193, 97-106.

21. Hac-Wydro, K. and Dynarowicz-Latka, P. 2006. Interaction between nystatin and natural membrane lipids in Langmuir monolayers-the role of a phospholipid in the mechanism of polyenes mode of action. Biophys. Chem. 123, 154-161.

22. Bharma, R., SaAd, A., Bolcsak, L.E., Janoff, A.S. and Swenson, C.E. 1997. Behavior of amphotericin B lipid complex in plasma in-vitro and in the circulation of rats. Antimcirob .Agents Chemother. 41, 886-892. 